\title{
Prospects for Vascular Access Education in Developing Countries: Current Situation in Cambodia
}

\author{
Toshihide Naganuma Yoshiaki Takemoto \\ Department of Urology, Osaka City University Graduate School of Medicine, Osaka, and Ubiquitous Blood \\ Purification International, Yokohama, Japan
}

\section{Keywords}

Cambodia $\cdot$ Dialysis history $\cdot$ Financial problems $\cdot$ Medical equipment and drugs - Medical training - Vascular access . Hemodialysis · Ubiquitous Blood Purification International

\begin{abstract}
We report our activities training doctors on vascular access procedures at International University (IU) Hospital in Cambodia through a program facilitated by Ubiquitous Blood Purification International, a nonprofit organization that provides medical support to developing countries in the field of dialysis medicine. Six doctors from Japan have been involved in the education of medical personnel at IU, and we have collectively visited Cambodia about 15 times from 2010 to 2016. In these visits, we have performed many operations, including 42 for arteriovenous fistula, 1 arteriovenous graft, and 1 percutaneous transluminal angioplasty. Stable development and management of vascular access is increasingly required in Cambodia due to increased use of dialysis therapy, and training of doctors in this technique is urgently required. However, we have encountered several difficulties that need to be addressed, including (1) the situation of personnel receiving this training, (2) problems with facilities, including medical equipment and drugs, (3) financial limitations, and (4) problems with management of vascular access.
\end{abstract}

(c) 2017 S. Karger AG, Basel

\section{KARGER}

(C) 2017 S. Karger AG, Basel

E-Mail karger@karger.com

www.karger.com/bpu

\section{Introduction}

The history of dialysis in Cambodia dates back to 1998, when a Chinese doctor opened a dialysis clinic in Phnom Penh. In 2001, this facility was moved to Calmette Hospital, Cambodia's flagship medical center. Before that time, end-stage renal failure patients had to go to $\mathrm{Ho} C h i$ Minh City (Vietnam) or Bangkok (Thailand) to receive dialysis treatment. After 2011, the number of dialysis centers increased temporarily to 10 , and in 2013 a center was established in Siem Reap, the first to open outside of Phnom Penh. Therefore, it has become possible for patients to receive dialysis treatment within Cambodia. Currently, however, there are only 7 centers open (at time of writing)because there is no national health insurance system and dialysis therapy must be paid for entirely by the patients themselves, making it difficult for the poor to receive this treatment. The average monthly wage in Cambodia is about USD 150, but the fee for 1 dialysis treatment is about USD 50. Therefore, patients who do not have enough money wait until they can save this

This article has already been published in: Contrib Nephrol. Basel, Karger, 2017, vol 189, pp 110-113.

Reproduced here with permission from the publisher.

These activities of blood access education were performed together with Yoshifumi Amari, Fumitaka Nakajima, Toru Hyodo, Nobuhisa Shibahara, and Kazunari Yoshida of NGO Ubiquitous Blood Purification International, Yokohama, Japan.

Toshihide Naganuma, MD, $\mathrm{PhD}$

Department of Urology, Osaka City University Medical School

1-4-3 Asahi-machi, Abeno-ku

Osaka 545-8585 (Japan)

E-Mail spxd48k9@ aria.ocn.ne.jp 


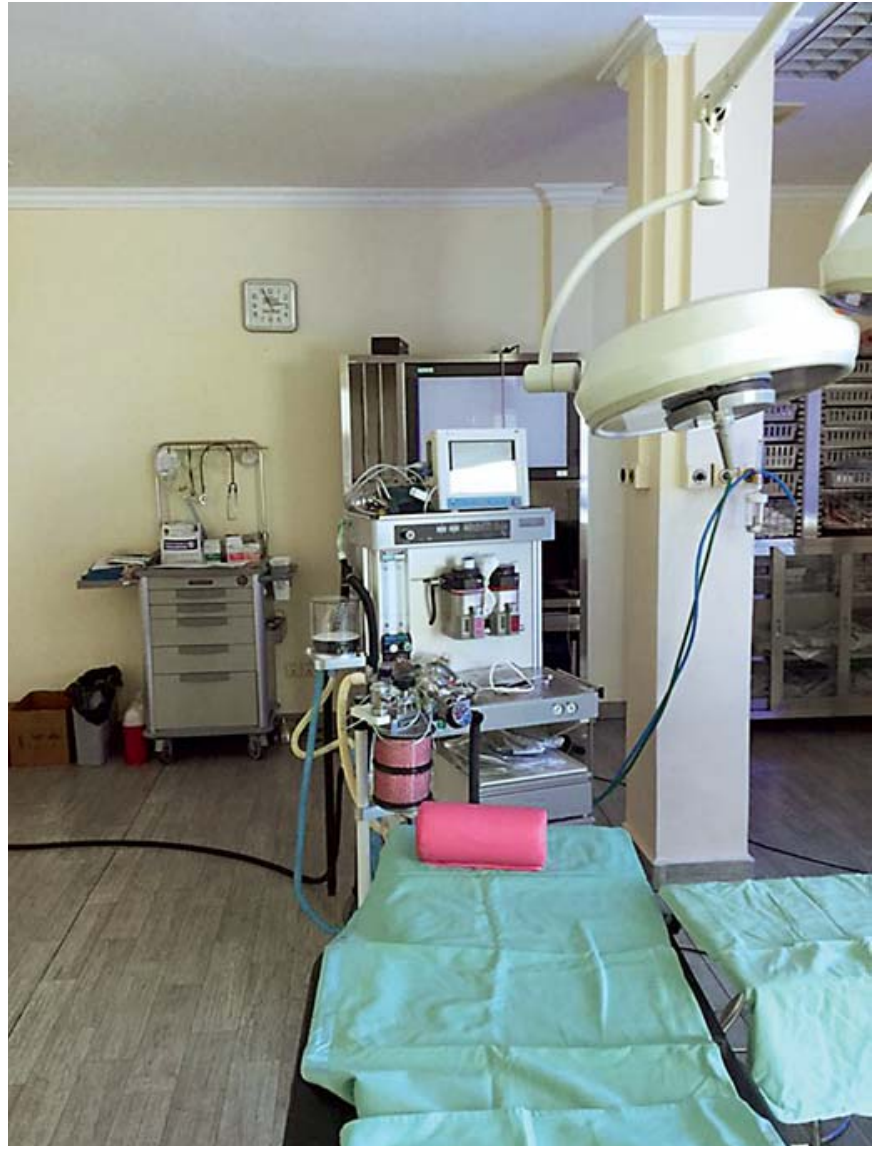

Fig. 1. Interior of the operating room. The room is equipped with the required anesthesia and lighting equipment.

amount, so that it is not rare for some patients to receive treatment once every 7 or 10 days. The total number of dialysis patients in Cambodia was 300-400 as of January 2016, with the primary disease being diabetes, followed by chronic glomerulonephritis. Peritoneal dialysis is not available because it is still not profitable for companies.

\section{Difficulties with Personnel Receiving Training}

In Cambodia, doctors who were mostly trained in France have typically performed arteriovenous fistula (AVF) procedures. In 2010, the Cambodia-Japan Friendship Blood Purification Center was opened through a donation to International University (IU) Hospital by the nonprofit organization Ubiquitous Blood Purification International (UBPI). Since then, we have provided various kinds of support to educate medical professionals in techniques of dialysis therapy [1]. Six doctors from Japan have been involved in the training of personnel at IU in vascu-

Prospects for Vascular Access Education in Developing Countries

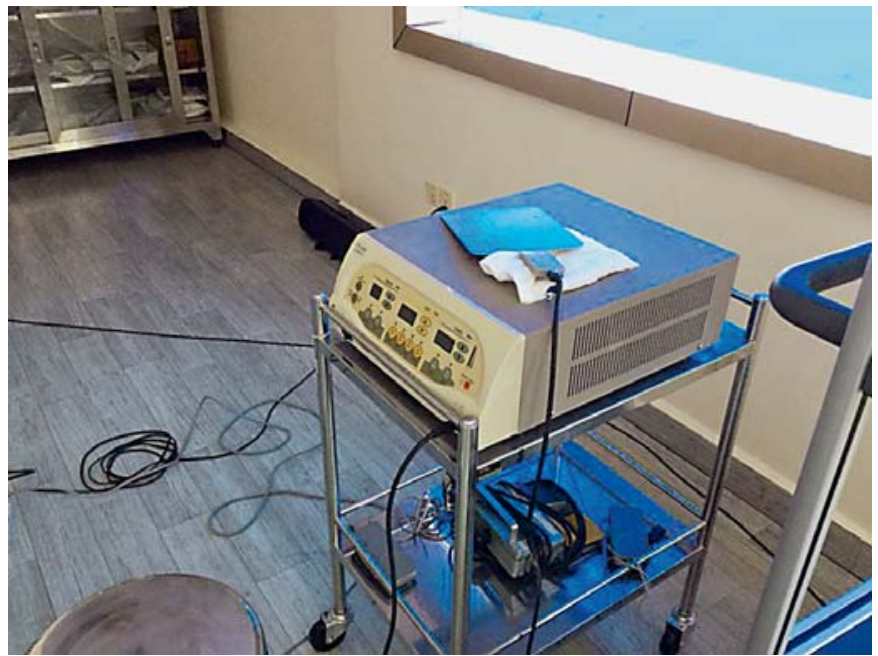

Fig. 2. Generator for monopolar and bipolar electrocautery.

lar access procedures, and we have visited Cambodia about 15 times from 2010 to 2016. During this time, we have performed 42 surgeries for AVF, 1 arteriovenous graft, and 1 percutaneous transluminal angioplasty (PTA). However, the doctors that we have trained have not remained at IU for long, and the techniques learned are not systematically passed on to other doctors. The personnel we taught have opened their own practices or moved to other hospitals. The doctor we are training now is from another country and was originally an otolaryngologist. Vascular access creation has become possible in simple virgin cases of AVF, but it is still difficult for cases with veins that are thin or require repair. First-generation doctors are now being trained, but our hope is for them to stay and go on to train second- and third-generation doctors.

\section{Difficulties with Medical Equipment and Drugs}

Basic medical equipment is mostly available, and the operating room is cleanly maintained and equipped with the necessary lighting. Equipment such as an ECG monitor, monopolar and bipolar electrocautery, fluoroscope, and an ultrasound machine are available (Fig. 1, 2). However, the inventory of supplies has not been managed properly and many AVF operation instruments that were available upon opening of the center are now missing. We have tried to restock these supplies by bringing items from Japan that were about to be disposed of or were donated by volunteers. Furthermore, supplies that are common in all operating rooms in Japan, such as silk suture and nylon suture with needles used for anastomosis, are

Blood Purif 2017;44(suppl 1):52-54 53 


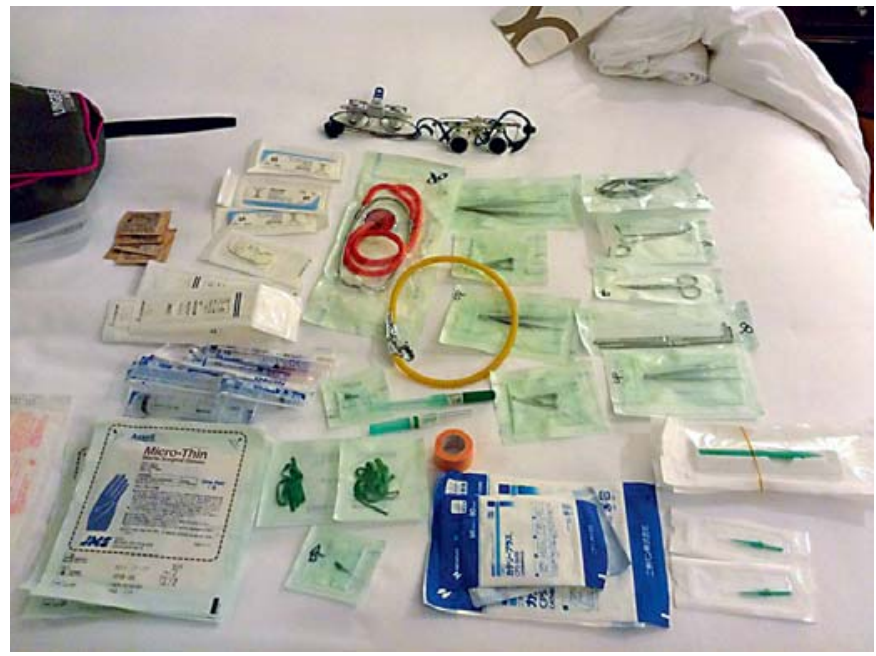

Fig. 3. Supplies brought from Japan.

unavailable due to the high cost and have to be brought from Japan (Fig. 3). Nylon suture with a cutting needle for skin closure is used as an alternative. Artificial blood vessels and PTA supplies are not available, as might be expected, and these also have to be brought from Japan.

\section{Financial Problems}

Because UBPI is a nonprofit organization, we have to pay for all travel expenses, and one trip to Cambodia costs about USD 4,000 each, including accommodation. A better arrangement is needed to make the technical support sustainable. Of course, it would also be better to have the hospital procure supplies such as silk suture and nylon suture for anastomosis. With regard to medical equipment used for artificial blood vessels and PTA, there is a need for the hospital to make an effort to buy them because there is a limit to what we can bring. Surgery fees are unclear and once we even performed a procedure for free. We are also aware of the country's circumstances, in which only the rich can afford this treatment due to the average monthly wage of USD 150 and lack of national health insurance, as mentioned above.

\section{Problems with Vascular Access Creation and Maintenance}

In addition to techniques not being systematically passed on due to trained doctors leaving the hospital, PTA cannot be used for stenosis and obstruction, in con-

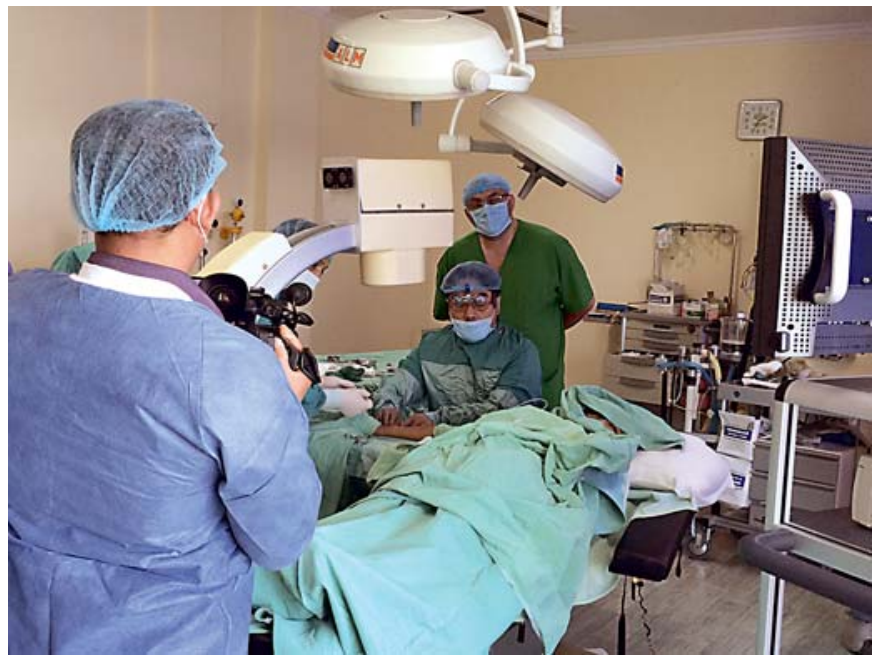

Fig. 4. First case of PTA performed in Cambodia.

trast to dialysis management in Japan. In such cases, surgical reconstruction is performed. In fact, we have witnessed some cases in which an AVF created at other hospitals was first located in the distal part of the forearm and then below the elbow, so that sooner or later AVF creation would not be possible in both arms. The PTA procedure performed this year by Dr. Naganuma was an obstruction case, and was the first PTA conducted in Cambodia (Fig. 4). As the percentage of television ownership is almost $100 \%$, we were able to publicize PTA as a procedure that can be used for AVF problems through a health education program of IU. There might be patients willing to pay for PTA on their own, but a governmental policy is required to make it available for all patients.

\section{Conclusion}

There are still many problems concerning the use of vascular access techniques in Cambodia. However, medical professionals in the country are highly motivated and this treatment needs to be available. Therefore, we will continue to find ways to achieve a sustainable medical support system for this purpose in Cambodia.

\section{Reference}

1 Naramura T, Hyodo T, Kokubo K, Matsubara H, Wakai H, Nakajima F, et al: Dialysis and quality of dialysate in Southeast Asian developing countries. Nephron Extra 2014;4:64-69.
54

Blood Purif 2017;44(suppl 1):52-54 DOI: $10.1159 / 000479620$
Naganuma/Takemoto 